\title{
Increased Serum Enzyme Levels Associated with Kupffer Cell Reduction with No Signs of Hepatic or Skeletal Muscle Injury
}

\author{
Zaher A. Radi, ${ }^{*}$ Petra H. Koza-Taylor, ${ }^{\dagger}$ \\ Rosonald R. Bell, ${ }^{\dagger}$ Leslie A. Obert, ${ }^{\dagger}$ \\ Herbert A. Runnels, ${ }^{\ddagger}$ Jean S. Beebe, ${ }^{*}$ \\ Michael P. Lawton, ${ }^{\dagger}$ and Seth Sadis ${ }^{\S}$ \\ From the Department of Biotherapeutics Research $\&$ \\ Development,* Pfizer Worldwide Research and Development, \\ Cambridge, Massachusetts; the Department of Drug Safety \\ Research and Development, ${ }^{\dagger}$ Pfizer Worldwide Research and \\ Development, Groton, Connecticut; the Department of Bioprocess \\ and Analytical, ${ }^{\ddagger}$ Pfizer Worldwide Research and Development, \\ Chesterfield, Missouri; and the Services Department, ${ }^{\S}$ Compendia \\ Bioscience, Ann Arbor, Michigan
}

Macrophage colony-stimulating factor (M-CSF) is a hematopoietic growth factor that is responsible for the survival and proliferation of monocytes and the differentiation of monocytes into macrophages, including Kupffer cells (KCs) in the liver. KCs play an important role in the clearance of several serum enzymes, including aspartate aminotransferase and creatine kinase, that are typically elevated as a result of liver or skeletal muscle injury. We used three distinct animal models to investigate the hypothesis that increases in the levels of serum enzymes can be the result of decreases in KCs in the apparent absence of hepatic or skeletal muscle injury. Specifically, neutralizing M-CSF activity via a novel human monoclonal antibody reduced the $\mathrm{CD} 14^{+} \mathrm{CD} 16^{+}$ monocyte population, depleted KCs, and increased aspartate aminotransferase and creatine kinase serum enzyme levels in cynomolgus macaques. In addition, the treatment of rats with clodronate liposomes depleted KCs and led to increased serum enzyme levels, again without evidence of tissue injury. Finally, in the osteopetrotic $\left(C s f 1^{o p} / C s f 1^{o p}\right)$ mice lacking functional M-CSF and having reduced levels of $\mathrm{KCs}$, the levels of serum enzymes are higher than in wild-type littermates. Together, these findings support a mechanism for increases in serum enzyme levels through M-CSF regulation of tissue macrophage homeostasis without concomitant histopathological changes in either the hepatic or skeletal system. (Am J Pathol 2011, 179:240-247; DOI: 10.1016/j.ajpath.2011.03.029)
Kupffer cells (KCs) are resident macrophages of the liver, predominantly distributed within the lumen of the hepatic sinusoids. ${ }^{1} \mathrm{KC}$ s are one of the largest tissue macrophage populations in the body and use phagocytosis, pinocytosis, and receptor-mediated endocytosis to filter blood of foreign and particulate material and maintain homeostasis of normal blood constituents. ${ }^{2-4}$ Receptors on KCs for Igs, immune complexes, and complement contribute to the liver's function as an important mediator of systemic and local innate immunity. In addition, a diverse array of cell surface receptors, including a large family of scavenger receptors, helps regulate lipid metabolism, assist in bactericidal functions, and promote removal of senescent or malformed red blood cells. Furthermore, surface receptors on KCs mediate the uptake of growth factors, cytokines, hormones, and enzymes. ${ }^{5,6}$ Unidentified surface receptors on KCs mediate the uptake of several short-lived serum enzymes, such as lactate dehydrogenase (LDH), creatine kinase (CK), and aspartate aminotransferase (AST). This process has been studied in vivo using radiolabeled derivatives of serum enzymes and in vitro using purified $\mathrm{KC}$ populations. ${ }^{7-9}$ Based on these results, it seems likely that modulators of KCs would disrupt normal pathways of serum enzyme clearance.

$\mathrm{KCs}$ originate from monocytes derived from the bone marrow through a differentiation program that normally requires the monocyte/macrophage colony-stimulating factor (M-CSF). ${ }^{10}$ Osteopetrotic (Csf1 $\left.{ }^{\mathrm{OP}} / \mathrm{Csf} 1^{\mathrm{OP}}\right)$ mice are defective in the production of CSF-1 (the murine isologue of human M-CSF) because of a point mutation in the coding region of the Csf1 gene and are deficient in several tissue macrophage populations, including $\mathrm{KCs}$, that are reduced to approximately $30 \%$ of the levels seen in wild-type littermates. ${ }^{11-13}$ Treatment of Csf10P/Csf1op mice with CSF-1 restores KC populations to their normal

Supported (and conducted) by Drug Safety Research and Development, Pfizer Worldwide Research and Development.

Accepted for publication March 15, 2011.

Disclosures: All authors have Pfizer stock options and were employed by Pfizer full time at the time the studies were conducted.

Address reprint request to Zaher A. Radi, D.V.M., Ph.D., Pfizer Worldwide Research and Development, Drug Safety Research and Development, 35 Cambridge Park Dr, Cambridge, MA 02140. E-mail: zaher.radi@pfizer.com. 
levels. ${ }^{14,15}$ Treatment of wild-type mice with anti-CSF-1 antibody has caused a similar decrease in KCs and other tissue macrophages. ${ }^{16}$ Therefore, at least in rodents, $\mathrm{M}$ CSF is important for KC differentiation and plays an important role in the maintenance and homeostasis of KCs. However, the role of M-CSF and KCs in nonhuman primates has not been investigated.

Antibodies to M-CSF have been characterized preclinically and clinically as potential treatments for autoimmune disease, cancer, and other illnesses. A single ascending-dose clinical trial using PD-0360324, a fully human IgG2 monoclonal antibody to M-CSF, was recently completed in healthy volunteers to evaluate safety, pharmacokinetics, and pharmacodynamics. ${ }^{17}$ During safety monitoring of this clinical study, dose-related increases in the levels of AST and CK were observed largely in the absence of other signals of hepatic and/or skeletal muscle injury. Therefore, the hypothesis that inhibition of M-CSF affected KC-mediated clearance of short-lived serum enzyme levels was considered.

The epitope recognized by PD-0360324 is conserved in human and nonhuman primate M-CSF but not in other animal species tested. To investigate the hypothesis that serum enzyme clearance is altered after $\mathrm{KC}$ reduction and depletion and to determine its relationship to hepatic and skeletal muscle pathophysiological features, we investigated the effects of PD-0360324 on KC and serum enzyme levels in an exploratory safety study using a nonhuman primate (cynomolgus macaque) model. Independently, we used dichloromethylene-bisphosphonate [clodronate (CLO)] liposomes to deplete KCs and to characterize the impact on several serum enzymes in a rat model. In this model, liposomes are ingested by macrophages via endocytosis and the phospholipid bilayers are disrupted by lysosomal phospholipases. The CLO that is released then accumulates in the macrophages and leads to cell death via apoptosis. ${ }^{18}$ This CLO model has been used extensively to study macrophages and in particular to assess the role that KCs play in modulating the inflammatory response observed in models of tissue injury. Examples include liver damage induced by acetaminophen, ${ }^{19},{ }^{20}$ lipopolysaccharide, ${ }^{21}$ and ethanol. ${ }^{22}$ Furthermore, we investigated serum enzyme levels in $C s f 1^{\circ D} / C s f 1^{\circ D}$ mice, another rodent model with decreased levels of KCs. For the first time, to our knowledge, we show in cynomolgus macaques and rodent models that depletion of KCs is associated with increased levels of short-lived serum enzymes.

\section{Materials and Methods}

\section{Animal Models and Experimental Design}

The animal care and experimental procedures for nonhuman primate (cynomolgus macaque), rat, and mouse studies were conducted in compliance with the US Animal Welfare Act and were performed in accordance with the standards of the Institute of Laboratory Animal Resources Guide. The Association for Assessment and Accreditation of Laboratory Animal Care International has accredited the Pfizer facility in which the studies were conducted.

\section{Experimental Design of the Dichloromethylene- Bisphosphonate KC Depletion Experiment in Rats}

In the dichloromethylene-bisphosphonate (CLO) liposome study, 6- to 7-week-old female Sprague-Dawley rats (weighing 100 to $150 \mathrm{~g}$ ) (Charles River Breeding Laboratories, Raleigh, NC) were used. Rats were housed individually in hanging polycarbonate cages in a single room dedicated to the study. The room environmental conditions had design specifications as follows: minimum of 12 air changes per hour with air filtered through $90 \%$ to 95\% efficiency filters and then through high-efficiency particulate air filters, relative humidity of $50 \% \pm 10 \%$ (humidity given as \pm raw \% values), temperature of $21^{\circ} \mathrm{C} \pm 3^{\circ} \mathrm{C}$, and a 12 -hour light-dark cycle. Food (Certified Rodent Diet 5002) and water (purified by reverse osmosis) were provided ad libitum. A CLO liposome suspension at a standard concentration of $7 \mathrm{mg} / \mathrm{mL}$ (http:// www.clodronateliposomes.org; last accessed May 31, 2011) in sterile PBS, a liposome suspension containing only PBS, was used for the control groups. Rats were randomly divided into two experimental groups: control and CLO treated. Body weights for all animals were determined before dosing. Seven rats per dose per group were i.v. injected once with either PBS or CLO (28 mg/kg) liposome suspensions (4 $\mu \mathrm{L} / \mathrm{g}$ body weight) in the tail vein. Predose blood samples (600 $\mu \mathrm{L}$ ) were collected by jugular venipuncture from each animal before liposome administration. In the CLO-treated rats, animals were observed daily for clinical signs and food consumption. Clinical chemistry parameters [alanine aminotransferase (ALT), AST, CK, glutamate dehydrogenase (GLDH), and LDH] were evaluated in samples collected before dosing and at 4,8 , 24, 48, 72, 96, and 192 hours after liposome administration. Animals were euthanized at 24, 72, and 192 hours after liposome administration. Liver and gastrocnemius and soleus muscles were formalin fixed at necropsy, stained by H\&E, and evaluated by light microscopy.

\section{Experimental Design of the CSF-1 Knockout Osteopetrotic Mouse Experiment}

In the knockout study, serum was obtained from male 1-month-old mice generated for a cross of hemizygous B6;C3Fe a/a-Csf $1^{\circ /} /+$ animals. All mice were genotyped at Jackson Laboratories, Bar Harbor, ME, to determine the allelic status of the Csf1 locus. Untreated Csf1op/ Csf1 ${ }^{\circ O}$ mice were euthanized, and blood was collected at necropsy. Serum samples from wild-type, heterozygous, and homozygous Csf1 ${ }^{\circ} / C s f 1^{\circ D}$ mice were analyzed for ALT, AST, CK, GLDH, and LDH levels.

\section{Experimental Design of the Anti-M-CSF Experiment in Nonhuman Primates}

In the drug PD-0360324 (an M-CSF-neutralizing human monoclonal antibody) safety study, female nonhuman primates (cynomolgus macaques, Macaca fascicularis), aged 3 to 5 years (weight, 3.2 to $4.1 \mathrm{~kg}$ ) (Charles River Laboratories, Wilmington, MA) were used. Animals were housed in a temperature- and humidity-monitored environment, with targeted temperature and relative humidity ranges of $18^{\circ} \mathrm{C}$ 
to $29^{\circ} \mathrm{C}$ and $30 \%$ to $70 \%$, respectively. Each assigned animal was implanted with a transponder (Biomedic Data Systems, Seaford, DE) that was mapped to a permanent study identification number. In compliance with the Animal Welfare Act, animals were compatible paired and remained in their designated stainless steel cages throughout the study. Water was provided ad libitum via an automatic system; a diet consisting of Lab Diet 5LR9 Certified HI-Fiber Primate Diet was provided twice daily and was supplemented with fresh fruit and/or vegetables. The drug, PD-0360324, was prepared using a stock solution of PD-0360324 that was refrigerated, prepared weekly in vials, and composed of $10.8 \mathrm{mg} / \mathrm{mL}$ in an L-histidine-buffered solution containing excipients at $\mathrm{pH}$ 6.0. The cynomolgus macaques were randomly divided into two experimental groups. Seven female monkeys per dose were treated with vehicle or $100 \mathrm{mg} / \mathrm{kg}$ PD-0360324 twice weekly for 29 days by the i.v. route of administration (by slow bolus at a dosing volume of approximately $9.26 \mathrm{~mL} / \mathrm{kg}$ over an approximate 2.5 -minute interval). The required volume of vehicle or drug solution for each animal was based on the most recent body weight. Animals were observed daily for clinical signs and food consumption. Physical examinations were conducted before testing and during the last week of dosing. Body weights for all animals were determined before testing; before dosing on days $1,8,15$, and 22; and on day 29, before necropsy. All clinical pathology parameters were analyzed using Advia and Olympus automated analyzers. Hematology parameters were evaluated in samples, containing EDTA as the anticoagulant, that were collected before testing and on day 29. In addition, ALT, AST, CK, and myoglobin levels were evaluated before testing, on each dosing day, and on day 29. Blood samples were collected from the femoral vein from all animals before testing and on the last day of treatment (approximately 6 hours after dosing) for measurement of PD-0360324 serum concentrations. Animals were euthanized on day 30 . Then, a complete necropsy was conducted on all animals, tissues were formalin fixed, and liver and various muscle sections (ie, diaphragm, gastrocnemius, soleus, extensor digitorum longus, psoas, and quadriceps) were stained with H\&E and evaluated by light microscopy.

\section{IHC and Laser-Scanning Cytometry}

In the PD-0360324-treated cynomolgus macaque study, KCs were quantitated in liver sections using CD163-staining laser-scanning cytometry. Liver tissue was fixed in 10\% neutral-buffered formalin for 24 hours and then paraffin embedded. Unstained sections (thickness, $3 \mu \mathrm{m}$ ) of the left and right lateral lobes of the livers from control and treated monkeys were placed on charged slides and air dried. Immediately before staining, the slides were placed in an oven at $60^{\circ} \mathrm{C}$ for approximately 10 minutes to enhance section adhesion. The Ventana Discovery autostainer was used for deparaffinization, heat-induced epitope retrieval, endogenous peroxidase quenching (4 minutes), and a 30minute blocking step in Serum Free Dako Blocking Serum (Dako, Carpinteria, CA). Slides were then rinsed in distilled water and transferred to a Dako autostainer for incubation with a KC marker (anti-human CD163 antibody; Santa Cruz Bio- technology, Inc., Santa Cruz, CA) at 1:150 dilution for 1 hour at room temperature, followed by incubation with goat antimouse lgG at 1:400 dilution for 45 minutes. This was followed by a 10-minute nuclear counterstain at 1:400 dilution. Slides were then removed from the stainer, and coverslips were applied with Prolong Gold mounting medium. A laser-scanning cytometer was used to evaluate the CD163 staining over the area of the liver comparing control with treated monkeys.

\section{IHC and Quantitative Image Analysis}

In the CLO-treated rat study, KCs in livers were quantitated using ED2 staining immunohistochemistry $(\mathrm{IHC})$ in liver sections obtained at 24, 72, and 192 hours after liposome administration. Portions of the left lateral liver lobe were fixed in 10\% neutral-buffered formalin for 24 hours and then paraffin embedded. Unstained sections (5- $\mu \mathrm{m}$ thick) were then cut and placed on charged slides and air dried. The Leica Autostainer XL was used for deparaffinization and hydration. Antigen retrieval was performed using Dako Proteinase K (Dako, Carpinteria, CA) for 2 minutes. Slides were then rinsed in distilled water and transferred to a Dako autostainer for endogenous peroxidase quenching (2 minutes). A 10-minute blocking step (two times) was performed in a Dako Biotin Blocking System, followed by 20 minutes in Dako Protein Block and incubation with ED2 (anti-rat CD163 antibody; Serotec, Raleigh, NC) at 1:100 dilution for 1 hour at room temperature. Finally, incubation was conducted with Vector biotinylated horse anti-mouse rat adsorbed IgG1 at a 1:150 dilution for 45 minutes. This was followed by 30-minute Vector Elite Avidin-Biotin Chromagin incubation. The slides were developed in Dako Liquid DAB + (Dako) for 5 minutes and counterstained with Mayer's hematoxylin (Dako), dehydrated, and covered with coverslips. For image analysis, a slide containing two sections of liver from each animal was imaged and captured on the Hamamatsu NanoZoomer virtual slide scanner (Olympus, Center Valley, PA) using the $\times 20$ objective setting. The images were collected and stored in Bacus Image Slide Format (Bacus Laboratories, Olympus). Virtual images were opened in Bacus WebSlide Enterprise software (Bacus Laboratories), and two images per section were captured at $\times 10$ objective virtual magnification from each section of liver and saved as .jpg images for ED2 stain quantification. Image Analysis images were opened in Image Pro-Plus (Media Cybernetics, Inc., Bethesda, MD). A macro was used to apply a $\times 10$ objective calibration to each of four images per animal. A value representing the percentage of stain area to tissue area for each image was collected, and a mean of percentage stain area was calculated for each animal.

\section{Statistical Analysis}

In the nonhuman primate safety study, statistical analysis of body weight data was conducted; a comparison of treated with control groups was performed on rank-transformed data using a $t$-test applied at two-tailed $1 \%$ and $5 \%$ significance. Analysis of AST, ALT, and CK was performed on rank-transformed data using a $t$-test applied at a one-tailed $1 \%$ and $5 \%$ significance level (testing for an 
increase in these parameters for the drug-treated group compared with the control group). Statistical analyses of quantitative $\mathrm{IHC}$ data in nonhuman primates were performed using repeated-measures analysis of variance, accounting for two experimental measures per animal (right and left lobes). In the rat CLO study, serum enzyme data were analyzed by comparing treated with control groups using a one-tailed Kruskal-Wallis test, applied at 1\% and 5\% significance. All parameters were analyzed separately for each point. Similarly, a one-tailed Kruskal-Wallis test was used to compare serum enzyme data from the wild-type, heterozygous, and homozygous $\mathrm{Csf1}$ op/Csf1 ${ }^{\circ \mathrm{P}}$ mice.

\section{Results}

No Changes in Clinical Signs, Physical

Examination Findings, Food Consumption, Body Weight, or Hematology Parameters, with the Exception of Monocytopenia, in the PD-0360324-Treated Cynomolgus Macaques

There were no treatment-related changes in clinical signs, physical examination findings, food consumption, body weight, or hematology parameters, with the exception of selective monocytopenia in the PD-0360324treated cynomolgus macaque group, that were similar to observations in previous toxicology studies with this drug and consistent with the effects of M-CSF on monocytes. Serum drug concentrations were evaluated at the end of the dosing period in animals receiving PD-0360324 and were increased and consistent with past experience at the $100 \mathrm{mg} / \mathrm{kg}$ dose level (data not shown). The circulating level of $\mathrm{CD} 14^{+} \mathrm{CD} 16^{+}$monocytes, a pharmacodynamic marker of the PD-0360324 mechanism of action, was evaluated in all animals. Consistent with the M-CSF expected pharmacological characteristics, this marker was maximally suppressed in the PD-0360324treated group but was not altered in the control group. Clinical chemistry parameters were unaffected, with the exception of PD-0360324-related increases in AST and CK.

\section{No Evidence of Liver or Muscle Injury but} Significant Reduction in KCs after PD-0360324

\section{Treatment}

A histopathological assessment of the liver and various skeletal muscle groups (ie, diaphragm, gastrocnemius, soleus, extensor digitorum longus, psoas, and quadriceps) did not reveal notable differences between the treatment and control groups. Liver sections were also evaluated by laser-scanning cytometry with antibodies to CD163, a macrophage marker. At the end of the dosing period, $\mathrm{CD}_{163^{+}}$cells in liver sections were significantly reduced in all animals receiving PD-0360324 (Figure 1). Treatment with PD-0360324 was associated with a twofold to threefold reduction in $\mathrm{CD}_{163^{+}}$(Kupffer) cells in the left and right lateral liver lobes compared with control animals.

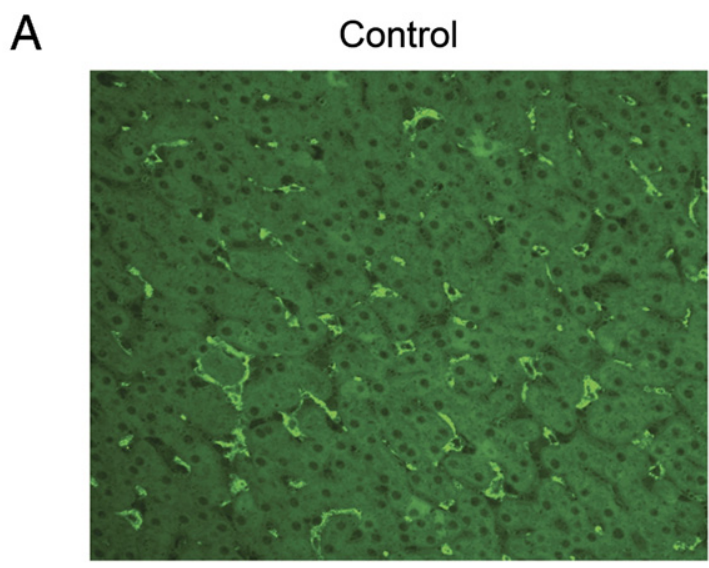

B

Treated

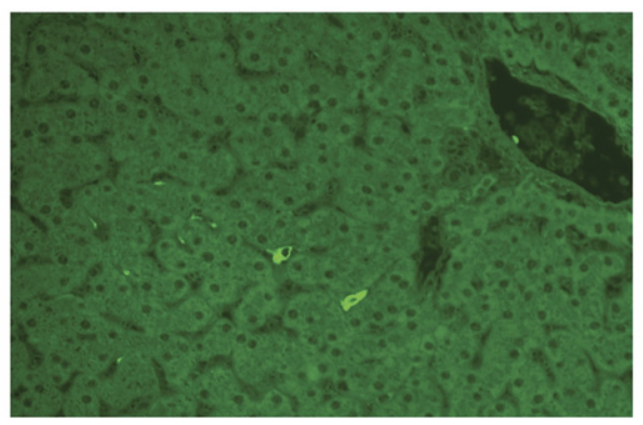

C Nonhuman Primate CD163 Laser Scanning Cytometry

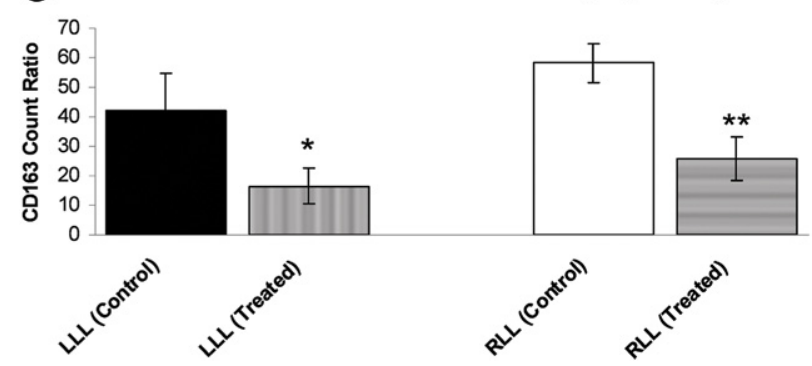

Figure 1. Nonhuman primate (cynomolgus macaque) liver section CD163 and KCs by laser-scanning cytometery (LSC). A: Liver section from a control monkey showing many scattered KCs. B: Liver section from a drug-treated monkey (the drug is a novel neutralizing human M-CSF monoclonal antibody) showing significantly fewer KCs. C: LSC quantitation. LLL indicates left lateral lobe; and RLL, right lateral lobe. There was a significant decrease in KCs in treated animals. ${ }^{*} P<0.05,{ }^{* *} P<0.01$.

\section{Significant Increases in AST and CK Enzyme Levels in Cynomolgus Macaques with PD-0360324 Treatment}

Treatment with PD-0360324 was associated with a twofold to threefold increase in the level of AST (Figure 2A) during the dosing period. In control animals, mean AST values did not appreciably change and ranged from 36 to $68 \mathrm{U} / \mathrm{L}$. In contrast, mean AST values in animals receiving PD-0360324 ranged from 76 to $141 \mathrm{U} / \mathrm{L}$ (Figure 2A). PD-0360324 treatment was also associated with an increase in the level of CK (Figure 2C); however, this parameter was associated with greater intra-animal and interanimal variability. In control animals, the mean CK 

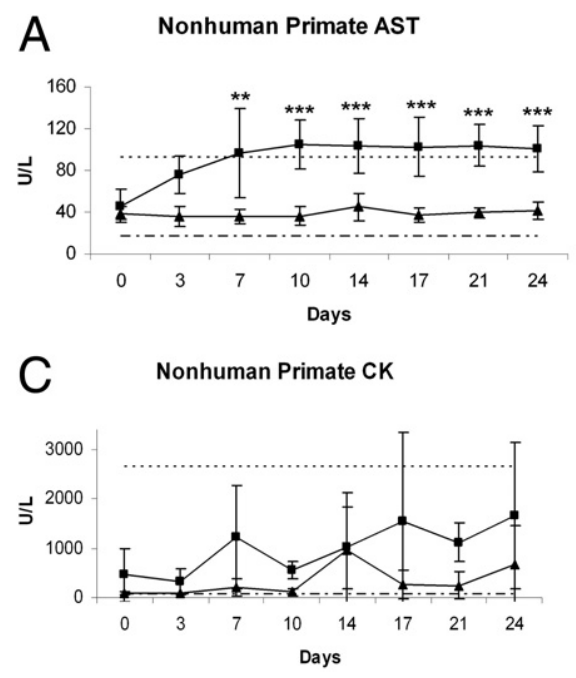

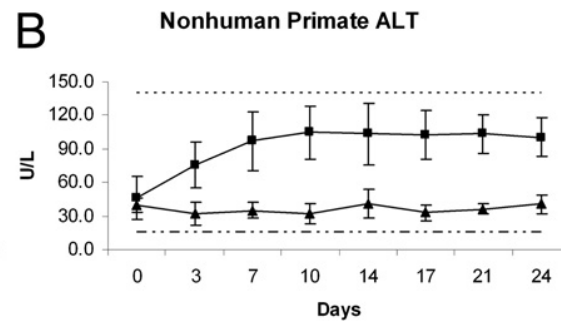

Donhuman Primate Myoglobin

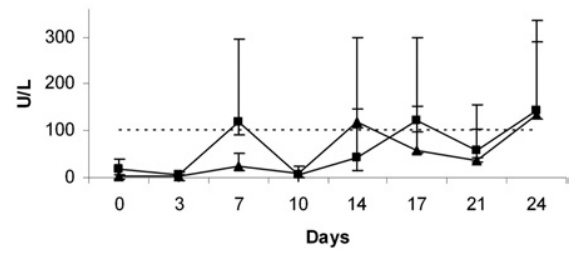

Figure 2. Clinical pathology serum enzyme levels in nonhuman primates (cynomolgus macaques) after treatment with a novel neutralizing human M-CSF monoclonal antibody: AST (A), ALT (B), CK (C), and myoglobin (D). A: There were significant increases in AST mean values after treatment with a novel neutralizing human M-CSF monoclonal antibody. B and D: ALT and myoglobin mean values, respectively, did not change. C: CK values show large variability. ${ }^{* * *} P \leq 0.05$, ${ }^{* * * * *} P \leq 0.01$ versus control. Triangles indicate control; squares indicate treated, dashed line indicates lower limit; and dotted line indicates upper limit. ranged from 78 to $2663 \mathrm{U} / \mathrm{L}$, whereas in animals receiving $100 \mathrm{mg} / \mathrm{kg}$ PD-0360324, the mean CK ranged from 332 to $7499 \mathrm{U} / \mathrm{L}$ (Figure 2C). In contrast to the treatment-related changes observed in AST and CK, no change was observed in the levels of serum ALT or myoglobin (Figure 2, $B$ and D). Although ALT levels did not reach statistical significance in PD-0360324-treated animals, there was an increase compared with controls.

\section{No Changes in Clinical Signs, Physical Examination Findings, Food Consumption, or Body Weight with CLO Treatment in Rats}

Next, we sought an independent method to deplete macrophages and characterize the effects on serum enzymes. Agents such as gadolinium chloride and CLO liposomes are effective at depleting macrophages in vivo and have been used in numerous studies ${ }^{19,23}$ to investigate the role of $\mathrm{KCs}$ in hepatic physiology. However, to our knowledge, the effects of these agents on serum enzyme levels have not been reported. Therefore, by using a rat model, we characterized the effect of CLO on $\mathrm{KCs}$ and investigated the effects of CLO-mediated KC depletion on several serum enzymes. Rats were divided into matched treatment groups and received a single i.v. injection of CLO $(28 \mathrm{mg} / \mathrm{kg})$ or PBS. There were no treatmentrelated changes in clinical signs, body weight, or food consumption parameters in rats treated with CLO or PBS.

\section{No Evidence of Liver or Muscle Injury but Significant Decreases in KCs after CLO Treatment in Rats}

Similarly, histopathological examination findings of the liver and muscle sections by light microscopy did not reveal significant differences between the treatment and control groups. To characterize the effects of CLO on $\mathrm{KCs}$ in rat liver, we performed morphometric analysis and IHC using the previously characterized monoclonal antibody ED2 macrophage marker. Rats treated with PBS

maintained normal levels of $\mathrm{ED}^{+}$cells in the liver throughout the experiment (Figure 3A). In contrast, rats given CLO experienced a rapid decrease in $\mathrm{ED}^{+}{ }^{+}$cells (Figure 3B), similar to previous reports. ${ }^{21}$ Liver sections from CLO-treated rats exhibited a marked decrease in ED2 ${ }^{+}$cells, observed 24 and 72 hours after treatment, and a moderate decrease by 192 hours (Figure 3C).

A
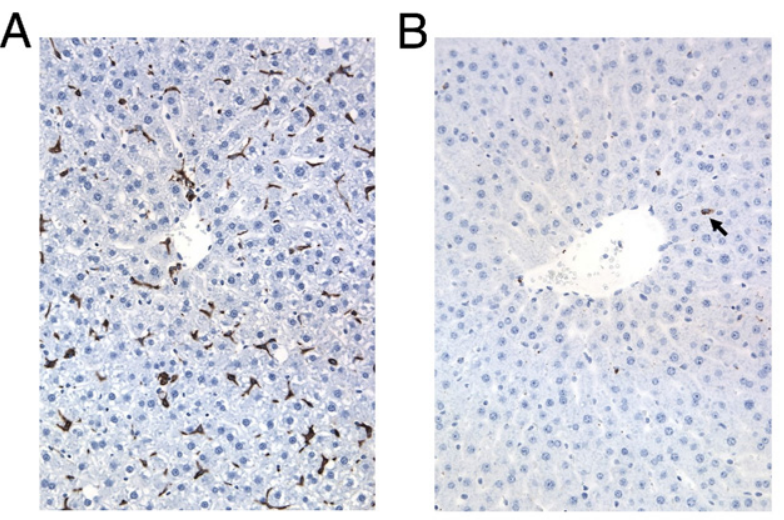

C

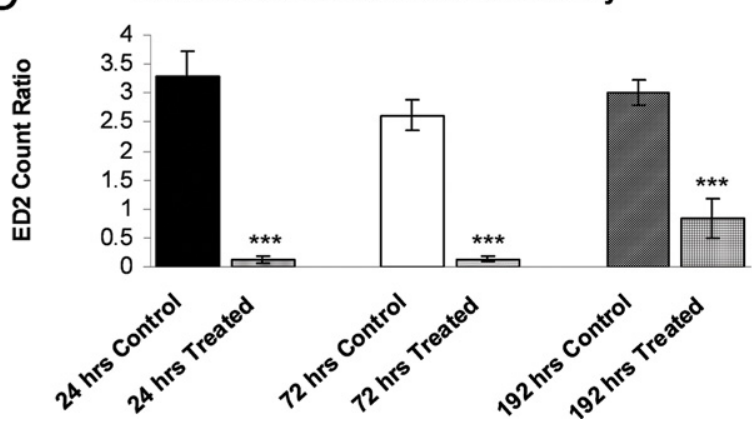

Figure 3. IHC of ED2 macrophage marker on liver sections obtained from rats that are either treated with PBS (A) or CLO liposomes (B) at 24 hours after treatment. There was a marked reduction in ED2 ${ }^{+}$cells in liver (arrow) from CLO-treated rats. Original magnification, $\times 20$. Image analysis of ED2 ratios (percentage of positive stained cells/total nuclei) for animals who underwent necropsy at 24, 72 , or 192 hours after CLO administration (C). There was a marked reduction in $\mathrm{ED} 2^{+}$cells at 24 and 72 hours and a moderate reduction at 192 hours. ${ }^{* * * *} P<0.0005$ versus control. 

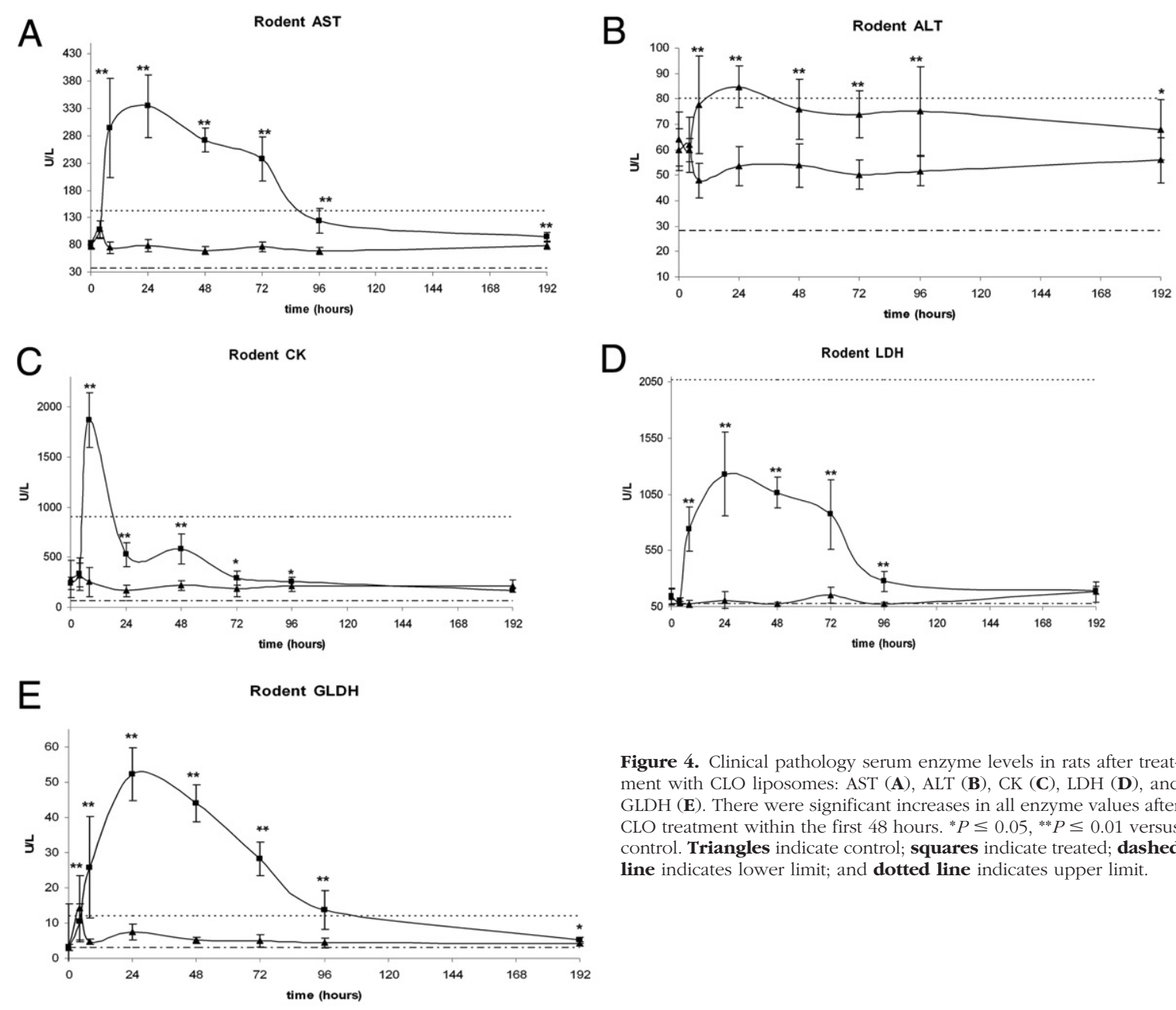

\section{Significant Increases in AST, CK, ALT, GLDH, and LDH Enzyme Levels in Rats with CLO}

\section{Treatment}

Blood samples were collected before dosing and at 4, 8, 24, 48, 72, 96, and 192 hours after treatment to investigate the effect of CLO-mediated KC depletion on serum levels of ALT, AST, CK, GLDH, and LDH. As expected, serum enzyme levels in rats treated with PBS were within normal limits. In contrast, the levels of AST, ALT, CK, GLDH, and $\mathrm{LDH}$ were increased after CLO treatment, reaching maximal levels within the first 2 days after treatment and then returning to normal levels by 192 hours (Figure 4, A-E). The greatest magnitude increase, relative to serum enzyme levels in control animals, was observed in LDH (Figure 4D), which exhibited a 25-fold increase in absolute value at 24 hours, remained elevated until 72 hours, and decreased markedly by 96 hours before returning to normal levels by 192 hours. Levels of GLDH (Figure 4E) and AST (Figure 4A) showed a similar transient pattern and attained 10 -fold and fourfold increases, respectively, relative to control levels at 24 hours. CK (Figure 4C) attained a maximal eightfold increase earlier than the other enzymes, at 8 hours after CLO treatment. In contrast to the temporal pattern exhibited by $\mathrm{LDH}, \mathrm{GLDH}, \mathrm{AST}$, and CK, the levels of ALT (Figure 4B)

Figure 4. Clinical pathology serum enzyme levels in rats after treatment with CLO liposomes: AST (A), ALT (B), CK (C), LDH (D), and $\mathrm{GLDH}(\mathbf{E})$. There were significant increases in all enzyme values after CLO treatment within the first 48 hours. ${ }^{*} P \leq 0.05,{ }^{* *} P \leq 0.01$ versus control. Triangles indicate control; squares indicate treated; dashed line indicates lower limit; and dotted line indicates upper limit.

experienced only a mild increase of approximately $50 \%$ at 24 hours after CLO treatment.

Significant Increases in AST, CK, ALT, GLDH, and LDH Enzyme Levels in the Csf1 ${ }^{\mathrm{OP}} / \mathrm{Csf} 1^{\mathrm{OP}}$ Knockout Mice

In the heterozygous knockout mice, only the level of ALT was slightly (30\%) elevated, and the levels of AST, CK, GLDH, and LDH were not significantly different from wildtype littermates (Table 1). The overall similarity between wild-type and heterozygous mice is consistent with the recessive nature of the CSF-1 gene. By comparison, all five serum enzymes analyzed were significantly increased in the homozygous mice compared with wild-type and heterozygous mice. The serum enzyme with the greatest increase in Csf1 $1^{\circ p} / C s f 1^{\circ p}$ mice was LDH (10-fold); the increase in the other serum enzymes ranged from threefold to sixfold.

\section{Discussion}

KCs represent a major tissue macrophage population with critical roles in innate immunity and the clearance of senescent or malformed red blood cells. ${ }^{5,6}$ Perhaps 
Table 1. Clinical Pathology Serum Enzyme Levels in Wild-Type, $\operatorname{Csf} 1^{o p} /+$, and $\operatorname{Cs} f 1^{o p} / \operatorname{Cs} f 1^{o p}$ Mice

\begin{tabular}{lccccc}
\hline & \multicolumn{5}{c}{ Serum enzymes (U/L) } \\
\cline { 2 - 5 } Phenotype & ALT & AST & CK & GLDH & LDH \\
\hline Wild type & $32.4 \pm 4.6$ & $61.7 \pm 17.5$ & $168.8 \pm 114.2$ & $11.5 \pm 1.9$ & $233.4 \pm 94.9$ \\
Heterozygous & $41 \pm 7^{*}$ & $70.6 \pm 13.2$ & $154 \pm 61.2$ & $14.6 \pm 5.5$ & $244 \pm 59.3$ \\
Homozygous & $113.8 \pm 45.3^{*}$ & $360.1 \pm 105.9^{*}$ & $506.5 \pm 303.1^{*}$ & $39.2 \pm 19.9^{*}$ & $2972.6 \pm 845.7^{*}$ \\
\hline
\end{tabular}

There were significant increases in all enzyme values in Csf1 ${ }^{\circ D} / C s f 1^{\circ D}$ mice.

*Significantly different from the wild type $(P \leq 0.01)$.

less widely appreciated is the role of KCs in regulating homeostasis of soluble bloodstream constituents, such as growth factors, hormones, and enzymes. In particular, the role that KCs play in maintaining homeostasis of serum enzymes and the in vivo effects of $\mathrm{KC}$ modulation on serum enzyme levels have not been well characterized. Herein, we present, for the first time to our knowledge, data showing that in vivo modulation of KCs is associated with kinetic changes in the levels of several short-lived serum enzymes not related to tissue injury.

The steady-state levels of short-lived enzymes are subject to rapid change after a significant change in either their production or their clearance. Several serum enzymes, such as ALT, AST, CK, and LDH, are widely used as indicators of tissue injury; the steady-state level of these enzymes is low in the healthy population but may increase markedly after injury to a specific tissue or organ, such as hepatic or skeletal muscle injury. Increases in CK and AST were observed in PD-0360324-treated monkeys. However, although these serum enzymes were elevated after $\mathrm{KC}$ reduction, either with an anti-M-CSF drug or by CLO administration, no histopathological evidence of either hepatic or skeletal muscle injury was observed in our studies in either nonhuman primates or rats. In addition, the levels of myoglobin (a muscle protein that increases in muscle injury) in drug-treated nonhuman primates were similar to those in controls.

Despite the increases in circulating CK and AST, increases in ALT levels did not reach statistical significance. This may be attributed to alternate routes of clearance for AST and ALT and/or different half-lives of the circulating enzymes. AST and ALT undergo endocytosis by nonparenchymal liver cells, including KCs and endothelial cells. ${ }^{24,25}$ Thus, it is possible that ALT is cleared predominately by endothelial cells, whereas the depletion of KCs has a more dramatic effect on AST, leading to the observed increase in circulating concentrations. The half-lives of serum enzymes vary widely, from a few hours to several days. Enzymes such as LDH, CK, and AST are short-lived and have halflives $<16$ hours, whereas the ALT half-life is estimated to be approximately 48 hours. ${ }^{26}$ Differences in half-life can have significance for clinical diagnosis. For example, differences in the stability of ALT and AST explain the distinct temporal pattern of enzyme accumulation after blunt trauma to the liver and assist in estimating when the trauma occurred. ${ }^{27}$

CD163 is a macrophage scavenger receptor expressed on most subpopulations of mature tissue macrophages, including $\mathrm{KCs}{ }^{28}$ The significant reduction in KCs (CD163 ${ }^{+}$cells) in liver sections from PD-0360324treated nonhuman primates confirmed the pharmacological effects of the drug on the tissue macrophage popu- lation. The initial observation in nonhuman primates that depletion of M-CSF leads to reductions in KCs and increases in serum enzymes is consistent with earlier reports $^{24,25,29}$ demonstrating a role for KCs in enzyme clearance. Similarly, ED2, which recognizes a membrane-bound antigen selectively expressed in the monocyte/macrophage lineage, ${ }^{30,31}$ was significantly decreased in CLO-treated rats. KCs are the cell population responsible for most serum enzyme clearance, as demonstrated by the research of Smit et $\mathrm{al}^{25}$ who showed that injection of radiolabeled enzymes resulted in the accumulation of radiolabel in the liver, spleen, and bone marrow. Subsequent cell fractionation experiments demonstrated that the radiolabel specifically localized to the macrophage cell population. ${ }^{25}$

In the 4-week study in cynomolgus macaques, treatment with PD-0360324 caused KC levels to decline to approximately $40 \%$ when compared with the level seen in vehicle-treated animals. This prolonged M-CSF neutralization was sufficient to attain a maximal effect on tissue macrophage populations because the effect of M-CSF withdrawal on $\mathrm{CD} 14^{+} \mathrm{CD} 16^{+}$monocytes reached steady state within 1 to 2 weeks. The marked reduction in KC levels is consistent with the effects of CSF-1 withdrawal in mouse models. ${ }^{14-16}$ Furthermore, $\mathrm{KC}$ depletion in rats and in nonhuman primates, coupled with the increase in serum enzymes without evidence of liver or skeletal muscle injury, is consistent with what has been observed in the $\mathrm{Csf1} 1^{\circ \mathrm{P}} / \mathrm{Csf1}^{\circ \mathrm{P}}$ mouse model, an osteopetrosis model with a defect in M-CSF production. No histopathological evidence of either hepatic or skeletal muscle injury was observed in the Csf1 ${ }^{O P} / C s f 1^{O P}$ mouse model. ${ }^{32}$ As noted earlier, Csf1 ${ }^{\text {OP }} / \mathrm{Csf1}^{\text {OP }}$ mice have only $30 \%$ of the level of KCs compared with wild-type mice. ${ }^{11}$ In these mice, our results show significant increases in multiple serum enzymes, consistent with serum enzyme increases observed in our nonhuman primate model. In nonhuman primates and rodents, M-CSF appears to be required to maintain tissue macrophage populations; other factors, perhaps granulocyte-M-CSF, function to maintain macrophage levels in the absence of M-CSF.

To further test the role of $\mathrm{KC}$ in enzyme clearance, we used a rodent model of KC depletion in which CLOencapsulated liposomes were injected i.v. and targeted KCs. As previously discussed, the CLO model has been used to study the role that KCs play in modulating the inflammatory response observed in models of tissue injury. The results presented herein demonstrate that this rodent model can also be used to study serum enzyme clearance. Although earlier publications have suggested a 
role for KCs in the clearance of $L D H, C K$, and AST, our findings demonstrate that serum GLDH levels are increased in KC-depleted rats, suggesting that this enzyme is also cleared by KCs. Further characterization of serum from animals treated with CLO liposomes could reveal additional blood constituents that are also cleared by KCs.

In summary, by using three distinct mechanisms of depleting KCs [ie, blockade of KC differentiation and/or survival via neutralization of M-CSF with PD-0360324 (nonhuman primates) or genetic deficiency of CSF (Csf1op/Csf1op mice) and ablation of resident KCs with CLO liposomes], we demonstrated that $\mathrm{KCs}$ regulate the steady-state levels and kinetics of several short-lived serum enzymes, including enzymes that are often used as indicators of hepatic and/or skeletal muscle injury during medical practice and clinical trials. Furthermore, the observation that increases in serum enzymes can be the result of a decrease in $\mathrm{KCs}$, and not a response to hepatic or muscle tissue injury, has broad implications for the interpretation of clinical chemistry data by providing an alternative explanation for the increases in serum enzymes that are normally considered markers of tissue injury.

\section{Acknowledgments}

We thank Richard Giovanelli for his technical assistance.

\section{References}

1. Naito M, Hasegawa G, Ebe $Y$, Yamamoto T: Differentiation and function of Kupffer cells. Med Electron Microsc 2004, 37:16-28

2. Charlier N, Neyrinck AM, Beghein N, Delzenne NM, Gallez B: Assessment of liver phagocytic activity using EPR spectrometry and imaging. Magn Reson Imaging 2009, 27:565-569

3. Helmy KY, Katschke KJ Jr, Gorgani NN, Kljavin NM, Elliott JM, Diehl L, Scales SJ, Ghilardi N, van Lookeren Campagne M: CRIg: a macrophage complement receptor required for phagocytosis of circulating pathogens. Cell 2006, 124:915-927

4. Toth CA, Thomas P: Liver endocytosis and Kupffer cells. Hepatology 1992, 16:255-266

5. Granado M, Martín Al, Priego T, Villanúa MA, López-Calderón A: Inactivation of Kupffer cells by gadolinium administration prevent lipopolysaccharide-induced decrease in liver insulin-like growth factor-I and IGF-binding protein-3 gene expression. J Endocrinol 2006, 188:503-511

6. Loegering DJ: Kupffer cell complement receptor clearance function and host defense. Circ Shock 1986, 20:321-333

7. Mahy BW, Rowson KE: Isoenzymic specificity of impaired clearance in mice infected with the riley virus. Science 1965, 149:756

8. Smit MJ, Beekhuis H, Duursma AM, Bouma JM, Gruber M: Catabolism of circulating enzymes: plasma clearance, endocytosis, and breakdown of lactate dehydrogenase-1 in rabbits. Clin Chem 1988, 34:2475-2480

9. Smit MJ, Duursma AM, Koudstaal J, Hardonk MJ, Bouma JM: Infection of mice with lactate dehydrogenase-elevating virus destroys the subpopulation of Kupffer cells involved in receptor-mediated endocytosis of lactate dehydrogenase and other enzymes. Hepatology 1990, 12:1192-1199

10. Stanley ER, Guilbert LJ, Tushinski RJ, Bartelmez SH: CSF-1: a mononuclear phagocyte lineage-specific hemopoietic growth factor. J Cell Biochem 1983, 21:151-159

11. Naito M, Hayashi S, Yoshida H, Nishikawa S, Shultz LD, Takahashi K: Abnormal differentiation of tissue macrophage populations in "osteopetrosis" (op) mice defective in the production of macrophage colony-stimulating factor. Am J Pathol 1991, 139:657-667

12. Yoshida H, Hayashi S, Kunisada T, Ogawa M, Nishikawa S, Okamura H, Sudo T, Shultz LD, Nishikawa S: The murine mutation osteopetrosis is in the coding region of the macrophage colony stimulating factor gene. Nature 1990, 345:442-444

13. Wiktor-Jedrzejczak W, Bartocci A, Ferrante AW Jr, Ahmed-Ansari A, Sell KW, Pollard JW, Stanley ER: Total absence of colony-stimulating factor 1 in the macrophage- deficient osteopetrotic (op/op) mouse. Proc Natl Acad Sci U S A 1990, 87:4828-4832

14. Cecchini MG, Dominguez MG, Mocci S, Wetterwald A, Felix R, Fleisch H, Chisholm O, Hofstetter W, Pollard JW, Stanley ER: Role of colony stimulating factor- 1 in the establishment and regulation of tissue macrophages during postnatal development of the mouse. Development 1994, 120:1357-1372

15. Yamamoto T, Kaizu C, Kawasaki T, Hasegawa G, Umezu H, Ohashi R, Sakurada J, Jiang S, Shultz L, Naito M: Macrophage colony-stimulating factor is indispensable for repopulation and differentiation of Kupffer cells but not for splenic red pulp macrophages in osteopetrotic (op/op) mice after macrophage depletion. Cell Tissue Res 2008, 332:245-256

16. Wei S, Lightwood D, Ladyman $H$, Cross $S$, Neale $H$, Griffiths $M$, Adams R, Marshall D, Lawson A, McKnight AJ, Stanley ER: Modulation of CSF-1-regulated post-natal development with anti-CSF-1 antibody. Immunobiology 2005, 210:109-119

17. Sadis S, Mukherjee A, Olson S, Dokmanovich M, Maher R, Cai C-H, Le V, Crawford M, Fedechko R, Whitfield L, Hellio Le Graverand Gastineau M-P, Stock T, Runnels HA, Zeiher B: Safety, pharmacokinetics, and pharmacodynamics of PD-0360324, a human monoclonal antibody to monocyte/macrophage colony stimulating factor, in healthy volunteers. Presented at the Annual Scientific Meeting of the American College of Rheumatology, October 17-21, 2009, Philadelphia, PA, Abstract 408

18. Van Rooijen N, Sanders A: Liposome mediated depletion of macrophages: mechanism of action, preparation of liposomes and applications. J Immunol Meth 1994, 174:83-93

19. Campion SN, Tatis-Rios C, Augustine LM, Goedken MJ, van Rooijen $\mathrm{N}$, Cherrington NJ, Manautou JE: Effect of allyl alcohol on hepatic transporter expression: zonal patterns of expression and role of Kupffer cell function. Toxicol Appl Pharmacol 2009, 236:49-58

20. Goldin RD, Ratnayaka ID, Breach CS, Brown IN, Wickramasinghe SN: Role of macrophages in acetaminophen (paracetamol)-induced hepatotoxicity. J Pathol 1996, 179:432-435

21. Sturm E, Havinga R, Baller JF, Wolters H, van Rooijen N, Kamps JA Verkade HJ, Karpen SJ, Kuipers F: Kupffer cell depletion with liposomal clodronate prevents suppression of Ntcp expression in endotoxin-treated rats. J Hepatol 2005, 42:102-109

22. Goldin RD, Ratnayaka ID, Brown IN, Wickramasinghe SN: The effect of Kupffer cell elimination on ethanol-induced liver damage in mice. Int J Exp Pathol 1995, 76:353-359

23. Korolenko TA, Klishevich MS, Cherkanova MS, Alexeenko TV, Zhanaeva SY, Savchenko NG, Goncharova IA, Filjushina EE: In vivo effect of selective macrophage suppression on the development of intrahepatic cholestasis in mice. Bull Exp Biol Med 2008, 146:396-400

24. Bijsterbosch MK, Duursma AM, Smit MJ, Bos OJ, Bouma JM, Gruber M: Several dehydrogenases and kinases compete for endocytosis from plasma by rat tissues. Biochem J 1985, 229:409-417

25. Smit MJ, Duursma AM, Joop M, Bouma W, Gruber JM: Receptor-mediated endocytosis of lactate dehydrogenase $M$, by liver macrophages: a mechanism for elimination of enzymes from plasma. J Biol Chem 1987, 262:13020-13026

26. Burtis CA, Ashwood ER (Eds): Tietz Textbook of Clinical Chemistry. Philadelphia, PA, WB Saunders \& Co, 1999

27. Kaku N: Short-term and long-term changes in hepatic function in 60 patients with blunt liver injury. J Trauma 1987, 27:607-614

28. Fabriek BO, Dijkstra CD, van den Berg TK: The macrophage scavenger receptor CD163. Immunobiology 2005, 210:153-160

29. De Jong AS, Duursma AM, Bourna JM, Gruber M, Brouwer A, Knook DL: Endocytosis of lactate dehydrogenase isoenzyme M4 in rats in vivo: experiments with enzyme labelled with O-(4-diazo-3,5di[125I]iodobenzoyl)sucrose. Biochem J 1982, 202:655-660

30. Dijkstra CD, Döpp EA, van den Berg TK, Damoiseaux JG: Monoclonal antibodies against rat macrophages. J Immunol Methods 1994, 174:21-23

31. Polfliet MM, Fabriek BO, Daniëls WP, Dijkstra CD, van den Berg TK: The rat macrophage scavenger receptor CD163: expression, regulation and role in inflammatory mediator production. Immunobiology 2006, 211:419-425

32. Radi ZA, Guzman RE, Bell RR: Increased connective tissue extracellular matrix in the op/op model of osteopetrosis. Pathobiology 2009, 76:199-203 